\title{
Fitz-Hugh-Curtis Syndrome
}

\author{
Tsukasa Nozu and Hiroaki Komiyama
}

Key words: Chlamydia trachomatis, Fitz-Hugh-Curtis Syndrome, perihepatitis

(DOI: 10.2169/internalmedicine.45.1645)

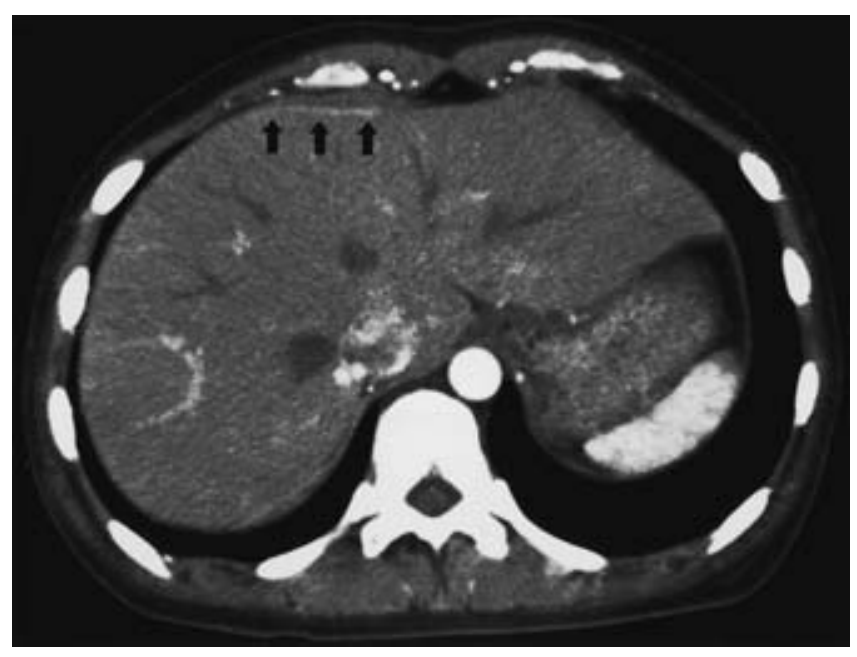

Figure 1. Fitz-Hugh-Curtis syndrome in a 25-year-old woman. Contrast-enhanced CT demonstrated marked hepatic capsular enhancement of the anterior surface of the right liver lobe (arrows, axial image).

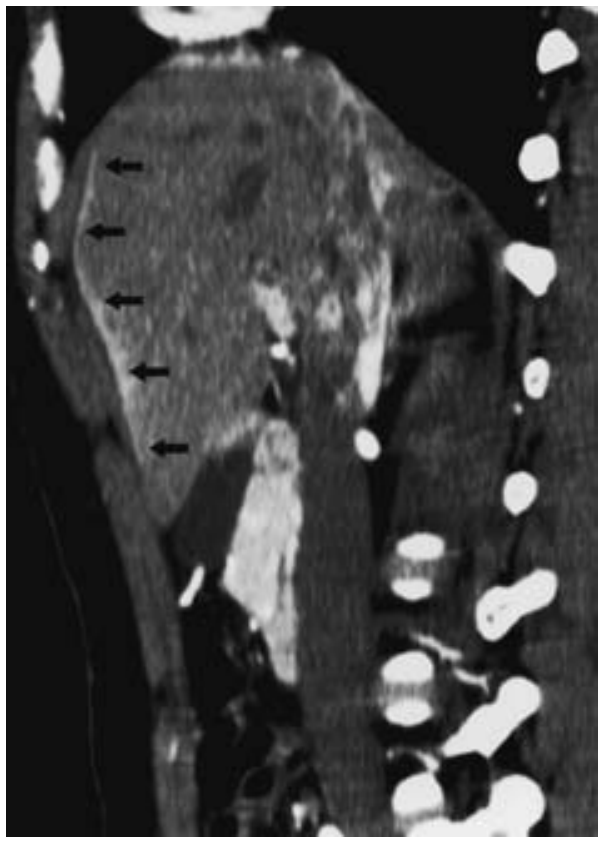

Figure 2. Sagittal image of the same patient. The hepatic capsular enhancement (arrows) was more visible in this image.

A 25-year-old woman who complained of severe right upper quadrant pain, was admitted to the hospital. Laboratory findings demonstrated slight leukocytosis and inflammatory reactions. Noncontrast computerized tomography (CT) of the abdomen demonstrated no abnormal findings. Then she underwent contrast-enhanced (CE) CT, which revealed marked hepatic capsular enhancement at arterial phase (Figs. 1, 2, arrows); however, it immediately disappeared and no abnormalities were seen in the later phase. The diagnosis of Fitz-Hugh-Curtis syndrome (FHCS) i.e., Chlamydia trachomatis perihepatitis, was confirmed by high titers of serum IgA and IgG antibody to Chlamydia trachomatis. She was treated with clarithromycin for 10 days, which resulted in complete resolution of her symptom.

The hepatic capsular enhancement on CECT has been reported to be useful for the early diagnosis of FHCS (1). As shown in the present case, multidetector-CT, which can generate multiple plane images with high resolution, is a highly sensitive imaging modality in the detection of this finding.

Department of Internal Medicine, Shari-Town General Hospital, Shari-Town

Received for publication November 9, 2005; Accepted for publication December 16, 2005

Correspondence to Tsukasa Nozu, Department of Internal Medicine, Shari-Town General Hospital, 41 Aoba-cho, Shari-Town, Shari-Gun, Hokkaido 099-4117 
DOI: 10.2169/internalmedicine.45.1645

\section{References}

1. Nishie A, Yoshimitsu K, Irie H, et al. Fitz-Hugh-Curtis syndrome. Radiologic manifestation. J Comput Assist Tomogr 27: 786-791, 2003.

(C) 2006 The Japanese Society of Internal Medicine http://www.naika.or.jp/imindex.html 